\title{
LA IDENTIDAD DE UNASUR: ¿REGIONALISMO POST-NEOLIBERAL O POST-HEGEMÓNICO?
}

\section{UNASUR'S IDENTITY: POST-NEOLIBERAL OR POST-HEGEMONIC REGIONALISM?}

\section{Nicolás Falomir Lockhart*}

\section{RESUMEN}

Desde comienzos del nuevo milenio se viene construyendo en América del Sur, un nuevo proceso de integración regional. Por sus particularidades, se trata de un proceso que desborda los modelos explicativos existentes. De allí la aparición de distintas propuestas de caracterización, como la del regionalismo post-neoliberal o la del post-hegemónico. Sin embargo, ninguna de ellas ha logrado aún una síntesis acabada del fenómeno sudamericano.

PALABRAS CLAVE: AMÉRICA DEL SUR * UNASUR * INTEGRACIÓN REGIONAL * REGIONALISMO

ABSTRACT

Since the new millennium, a new process of regional integration is being built in South America. However, due to its particularities it is a process that cannot be explained by the pre-existing models of integration. Therefore, many conceptualizations have been proposed, such as post-neoliberal regionalism or post-hegemonic regionalism. Yet, none of them has reached a successful synthesis of the South American phenomenon.

KEYWORDS: SOUTH AMERICA * UNASUR * REGIONAL INTEGRATION * REGIONALISM

Cátedra de Derecho Internacional Público e Instituto de Relaciones Internacionales (IRI), ambos de la Universidad Nacional de La Plata (UNLP), Argentina.

nfalomir@gmail.com 


\section{INTRODUCCIÓN}

En América del Sur, a partir del comienzo del nuevo milenio, se viene construyendo una nueva experiencia de integración regional llamada UNASUR (Unión de Naciones Suramericanas, desde el 2007). Por sus características novedosas no es posible encuadrarla en ninguno de los modelos explicativos precedentes. Esto ha generado un debate sobre la naturaleza de tal proceso, al punto que algunos autores ponen en duda su carácter de fenómeno de integración y lo catalogan como una mera experiencia de cooperación o coordinación. Más aún, se afirma que por distracción de esfuerzos, atenta contra los objetivos de la integración propuesta durante los años 90 . De allí que se le señale como factor de divergencia y fragmentación regional (Bouzas, 2008).

Por otra parte, se entiende que la UNASUR es un verdadero proceso de integración regional, que desborda los modelos de los años 90 y que por ello, amerita que pensemos nuevas categorías conceptuales para comprenderlo. Aunque ha habido intentos, se cree que no se ha logrado una síntesis acabada del fenómeno. En este artículo, luego de analizar la influencia del "regionalismo abierto" en América Latina hasta el nuevo milenio, se expondrán los aciertos y críticas de dos definiciones recientemente propuestas: la del "regionalismo post-neoliberal" y la del "regionalismo post-hegemónico". Finalmente, se plantea una propuesta de caracterización como "regionalismo sudamericano". Con ello no se pretende agotar el debate, pero sí hacer un modesto aporte al desafío de comprender la naturaleza de la UNASUR.

\section{DEL REGIONALISMO ABIERTO A LA BÚSQUEDA DE UN NUEVO MODELO DE INTEGRACIÓN}

A diferencia de lo sucedido en Europa, escenario de la Segunda Guerra Mundial, donde el proceso de integración surgió como un mecanismo para asegurar la paz (Haas, 1948), en América Latina, el objetivo de la integración regional siempre ha sido el desarrollo económico (Prebisch, 1948). Según el pensamiento cepalino, para que los países de la región se desarrollaran, tenían que superar el deterioro en los términos de intercambio y para ello, debían llevar adelante una industrialización por sustitución de importaciones. La asociación con los vecinos tenía por fin generar un mercado interno ampliado (un mercado regional) capaz de sostener la demanda de esos productos con valor agregado. Esa inspiración la tuvieron el Mercado Común de Centro América (MCCA) y el Grupo Andino (en su primera versión).

Sin perjuicio de esas experiencias, surgieron otras concebidas al abrigo del liberalismo económico, como la Asociación Latinoamericana de Libre Comercio (ALALC). En estas, el Estado no tenía un rol activo de planificación en la economía, mucho menos de productor. El desarrollo vendría de la mano del crecimiento de las exportaciones y el Estado se limitaba a estimular a estas últimas a través de la reducción arancelaria. Este fue el esquema que se fue imponiendo en América Latina hasta llegar a ser en los 90, el modelo por antonomasia.

Desde entonces, los latinoamericanos nos "acostumbramos" a pensar que la única integración regional posible era la económica y a través de la eliminación de las barreras arancelarias y la desregulación para el "libre" movimiento de capitales. Un proceso cuya conducción -en sintonía con los postulados del "Consenso de Washington"- era cedida a las fuerzas del mercado ${ }^{1}$. Su influencia fue tal, que moldeó los esquemas que se formaron en esos años como el Mercosur, generando la reformulación en los objetivos de los esquemas pre existentes, caso del Pacto Andino (Comunidad Andina desde 1997), adecuándolos a la globalización económico-financiera (Molano, 2011: 38).

Es importante tener presente la realidad de los países de la región a comienzos de los 90. La Guerra Fría había concluido y con ella, la importancia estratégica de América Latina en el contexto bipolar (Rojas, 2008-2009: 100 y Lowenthal, 2006: 74). Por otra parte, el relajamiento de las tensiones Este-Oeste trajo nuevas posibilidades de acción y un renovado interés por la cooperación internacional (Hurrell, 1995: 18-21) que los latinoamericanos veían como la

1 La noción de "regionalismo abierto" pretendía marcar distancia de las experiencias previas de industrialización por sustitución de importaciones. La fórmula ganadora que se repetía entonces era: export-led growth y su estímulo se lograba con desregulación y liberalización comercial. 
oportunidad para superar el legado de la "década perdida" de los años 80. El temor de quedar al margen de la mundialización económicofinanciera, los condujo a asociarse para ganar competitividad internacional y lo hicieron abrazando el regionalismo abierto como única opción válida de inserción internacional. Como señaló oportunamente la Comisión Económica para América Latina y El Caribe (CEPAL) (1994: 8), se trató de una estrategia defensiva, para reducir su vulnerabilidad en la nueva economía globalizada (Soares de Lima, 2006: 3)2 .

Más allá del consenso señalado, había entre los latinoamericanos diferencias en las estrategias de inserción comercial (León, 2000: 230 y Sanahuja, 2008: 76). De allí, que los años 90 hayan dejado en América Latina un complejo de subregiones, con bloques de integración y proyectos políticos distintos. Situación interpretada como "fragmentación regional" por las diferencias y distancias que se generaron entre las distintas subregiones (Bouzas, 2008: 320$)^{3}$. América Latina, entonces, se desagregó ${ }^{4}$ y ello impedía identificarla como un espacio homogéneo ${ }^{5}$.

$2 \quad$ El problema era que el modelo solo era viable con acceso estable y seguro a los mercados de los países desarrollados que practicaban el proteccionismo para con los bienes en los que los latinoamericanos eran especialmente competitivos. De allí, la profundización de la vulnerabilidad económica y del perfil exportador de materias primas de América Latina (Kacowicz, 2008: 116).

3 Para finales del siglo XX, la imagen de una América Latina más o menos homogénea había cedido a la de un mosaico de subregiones. México integrado a América del Norte (González, 2008: 122), incluso en lo financiero, tecnológico, militar y cada vez más en lo cultural (Tokatlian, 2001: 143). Solo en Sudamérica se destacaban los siguientes bloques: el Mercosur, la CAN, el CARICOM — por Surinam y Guyana-y el modelo de apertura al mundo o trading around de Chile (Smith, 2000: 325).

Es ilustrativa la presencia comercial de EEUU en las distintas subregiones. A comienzos del 2000, EEUU participaba en el 74\% del mercado mexicano, $48 \%$ del centroamericano y $40 \%$ del caribeño. El índice bajaba al $35 \%$ en la CAN y a $21 \%$ en el Mercosur (Carranza, 2000: 122). Las relaciones entre EEUU y América Latina son la suma de relaciones con cada subregión (Lowenthal, 2006: 75).

Es significativo que un número especial de la revista Foreign Affairs en Español (2006) se
El regionalismo abierto demostró ser muy eficaz para la eliminación de las barreras al comercio (quintuplicándose el comercio intrarregional entre 1990 y 2005 (Sanahuja, 2006: 86)). No obstante, tal eficacia no se vio reflejada en cuestiones que también hacen a la integración como: la concreción de una política comercial común ante terceros, estándares comunes de estabilidad macroeconómica, convergencia en políticas sectoriales estratégicas (como en transporte, energía o innovación tecnológica), reducción de asimetrías o la construcción de un marco normativo-institucional que garantizara el gobierno del proceso integrador.

Por otra parte, el éxito de ese tipo de integración fue funcional a la erosión de las capacidades de los Estados para resolver los problemas de la ciudadanía (Rojas, 2006: 115). En pos de "dejar espacio de crecimiento" al mercado, el Estado se replegó al punto de tolerar altos grados de exclusión social (Panizza, 2009 y Stiglitz, 2006: 32-34). La integración de los 90 , solo beneficiaba a los sectores vinculados con la exportación de materias primas y al financiero. Su contracara era la descomposición del tejido social, reflejado en alarmantes tasas de desigualdad social, pobreza e indigencia, en el debilitamiento de las instituciones democráticas y el desapego a tal sistema de gobierno (Rojas, 2008: 8).

Esos efectos negativos fueron sentando las bases de un consenso - relativamente general- sobre el agotamiento de tal modelo. La respuesta fue una propuesta integradora con nuevo perfil, elaborada en torno a una agenda de desarrollo donde el Estado recuperaba su rol activo (CEPAL, 2004: 84). Así, Latinoamérica entró en una nueva fase de integración regional ${ }^{6}$ cuyos rasgos son: la recuperación

haya destinado a este tema bajo el título "¿Existe América Latina?” (Vol. 6 nro. 1, enero 2006).

6 Lagos y Zovatto (2007: 11) entienden que el proceso de integración latinoamericana puede ser dividido en tres etapas : la voluntarista - década del 50 hasta la primera de los 70 - , la revisionista —de la segunda mitad de los 70 hasta fines de los 80 - y la pragmática — propia de los años 90 . Continuando con esa idea, desde comienzos del nuevo milenio sería posible identificar una cuarta etapa (política y de desarrollo). 
de la conducción del Estado y la complementación de aquella liberalización comercial con el estímulo a las áreas relegadas, con especial celo en el desarrollo y la integración física. Se trata de un modelo con objetivos múltiples: la promoción de las capacidades institucionales, la concertación de políticas comunes, la integración estratégico-sectorial, la integración física, la participación social, el tratamiento de las asimetrías y la promoción del desarrollo en general (CEPAL, 2004: 84). De allí, el carácter multidimensional de esta nueva fase (Sanahuja, 2011: 118).

Es importante señalar ciertas diferencias entre este nuevo tipo de integración y el regionalismo abierto. La primera, refiere al rol activo del Estado y su dirección del proceso integrador, poniendo a la política en el centro de la escena. En este es el Estado quien aporta la visión estratégica y define el perfil de la integración. La segunda refiere al contenido multidimensional por oposición a lo meramente comercial. Otra refiere a la concepción que se le da a los territorios nacionales: ya no son espacios en los que es necesario borrar las fronteras para asegurar el flujo comercial "normal", sino que son una "contigüidad geográfica"; una condición de existencia. La idea de integración física y productiva transnacional da a este esquema un sentido de permanencia más sustentable que la mera asociación por liberalización arancelaria (Soares de Lima, 2006: 4-6; 2008: 24) . Por último, cabe distinguir el alcance sudamericano, superando la lógica subregional de los 90 (Álvarez, 2009: 6). Cabe aclarar, sin embargo, que tales esquemas subregionales no desaparecieron sino que fueron comprendidos en el nuevo proceso. En efecto, la UNASUR reconoce su existencia y objetivos, asimismo se sirve de su institucionalidad (CSN, 2004).

$7 \quad$ Esta autora sostiene que la integración física y productiva tiende a superar el "síndrome colonial" de la desconexión, según el cual cada unidad política solo se comunicaba con sus vecinos a través de metrópoli (2006: 5). Como un bien común (regional), una vez construida esa infraestructura de integración, nadie puede ser excluido de su disfrute, lo que repercute a nivel local, nacional y regional, más allá de los operadores comerciales que se ven beneficiados con vías más ágiles y baratas.
Ahora bien, cómo calificar este novel proceso de integración que se da con un nuevo marco geográfico y con características nuevas. A continuación, se abocará en el análisis de los aciertos y falencias de dos propuestas: la conceptualización como regionalismo post-neoliberal y la del regionalismo post-hegemónico.

\section{LA INTEGRACIÓN SUDAMERICANA COMO PROCESO POST-NEOLIBERAL}

Dada la trascendencia de los límites geográficos subregionales y del contenido exclusivamente comercial de los 90, algunos autores como Grugel y Riggirozzi (2012), Rovira Katwasser (2011) o McDonald y Rückert (2009), han caracterizado a esta experiencia sudamericana como "regionalismo post-neoliberal". Otros, como Sanahuja (2008), la llaman "integración post-liberal"; aunque se trata de una denominación menos usada. Ambos casos refieren al conjunto de características que diferencia a esa nueva fase del regionalismo con el modelo vigente hasta entonces.

Si bien, este nuevo modelo se sigue apoyando en el crecimiento a través de las exportaciones $-y$ en el celo del equilibrio fiscal-, incorpora a la fórmula de desarrollo "la vuelta al Estado". La recuperación de las capacidades estatales, como lógica reacción a la excesiva presencia del mercado, se justifica en la necesidad de establecer mecanismos de inclusión social. El post-neoliberalismo refleja el consenso sobre la necesidad de adopción de medidas como: la dirección de la inversión pública, el manejo de las exportaciones, la redistribución de la riqueza, el incentivo al consumo interno o la adopción de medidas contra-cíclicas. Se destaca, entonces, la conducción política orientada hacia el bienestar de la sociedad, por oposición a la conducción "tecnocrática" del regionalismo abierto.

En el caso de la UNASUR, esa orientación se ve en lo que aquí se señala como el rasgo multidimensional. Aún cuando incluye entre sus metas la conformación de un área de libre comercio en América del Sur, encara la integración económica desde la perspectiva del desarrollo a través de la integración productiva. La iniciativa IIRSA (Integración de la Infraestructura Regional en América del Sur) representa un claro ejemplo de ello y 
evidencia la nueva concepción del subcontinente como "un espacio geo-económico plenamente integrado" (IIRSA, 2011). Según esta idea, la integración y el desarrollo van de la mano; de allí los diez ejes de integración y desarrollo diseñados en franjas multinacionales que concentran flujos de comercio, actuales $y$ potenciales, dotándolos de transporte, energía y comunicaciones, promoviendo cadenas productivas - que pudieran generar valor agregado-, con economías de escala a lo largo de esos ejes.

La iniciativa se completa con un plan de estímulo a las exportaciones. En la I Reunión de Jefes de Estado de la Comunidad Sudamericana de Naciones (CSN) realizada en Brasilia, en 2005, se adoptó dos mecanismos de promoción económica: 1) la creación gradual de un área de libre comercio sudamericana, con base en la convergencia entre el Mercosur y la Comunidad Andina, y 2) la promoción de las exportaciones sobre la base del modelo PSCI (Programa de Sustitución Competitiva de Importaciones) de Brasil. El programa se creó para disminuir las asimetrías comerciales con los vecinos a través de la sustitución de productos provenientes de América del Norte, Europa o Asia por productos provenientes de países sudamericanos (Mallmann, 2007: 53); pero también contemplaba la posibilidad de asociación de su sector privado con los empresarios brasileños a fin de aprovechar su logística y apoyo financiero para lograr articular ventas de productos sudamericanos al mundo (PSCI, 2011).

En definitiva, tanto la iniciativa IIRSA como el plan de estímulo a las exportaciones ponen de manifiesto una visión estratégica sudamericana (Álvarez, 2009: 2). Planteada desde la génesis en el año 2000, esa visión se ha ido consolidando hasta el punto de institucionalizarse como lo prueba la creación del Cosiplan (Consejo Sudamericano de Infraestructura y Planeamiento) en 2009, durante la III Reunión Ordinaria del Consejo de Jefas $y$ Jefes de la UNASUR. Su estatuto incorpora como propios los objetivos, los planes y la visión de la iniciativa IIRSA. Además, define como instancia de apoyo a su Foro Técnico para dicha empresa.
Otra dimensión comprendida por este nuevo regionalismo post-neoliberal es la financiera. Desde el Consenso de Guayaquil en 2002 - luego de las crisis financieras argentinas y uruguayas - se identificó como necesidad, la reducción de la vulnerabilidad internacional de los países de la región, por ejemplo, a través de mecanismos de solidaridad financiera con la democracia y la gobernabilidad. También se reconoció su importancia a los fines de garantizar la ejecución de la Agenda Social y la de Integración Productiva en América del Sur. De allí la idea, discutida en la Cumbre de Cochabamba de 2006, de crear un Sistema Financiero Sudamericano con un Sistema Multilateral de Pagos en Monedas Locales, el desarrollo de un Mercado de Deuda Regional, la constitución de un Fondo de Estabilización Macroeconómica (Fondo de Reserva) - a fin de prevenir los flujos de capital especulativos - y la creación del Banco del Sur.

Otras dimensiones de la nueva agenda de la integración son, por ejemplo, el desarrollo social, que motivó la creación del Consejo de Desarrollo Social Sudamericano (CDSS) como espacio de diálogo, cooperación y coordinación para lograr el desarrollo social y humano integral de los pueblos sudamericanos. O la nueva agenda regional sobre el tratamiento coordinado de la droga, gestionado en el seno del Consejo Sudamericano sobre el Problema Mundial de la Droga.

Un último caso de relevancia que sería oportuno señalar, es la integración energética, parcialmente contemplada en la iniciativa IIRSA. Atendiendo a su riqueza, se propuso interconectar la región a fin de satisfacer las demandas energéticas del desarrollo de los sudamericanos, reafirmando la visión estratégico-regional. Más allá de la creación del Consejo Energético Sudamericano y de las sucesivas declaraciones a favor de adoptar un Tratado Energético Sudamericano, es de esperar que el juego de intereses de los miembros de UNASUR (la dinámica interna del proceso integrador) complejice la integración energética sudamericana.

En suma, la calificación de la integración sudamericana como proceso post-neoliberal parece adecuada para explicar la trascendencia del modelo neoliberal, con la dirección estatal 
en el proceso económico como dato distintivo. Su acción se orienta en pos de una mayor inclusión social y del combate a la pobreza, mitigando los efectos negativos de la integración neoliberal, de desregulación económica y excesiva permisión al mercado. Sin embargo, se entiende que esa caracterización deja entrever la superación de las políticas neoliberales cuando en realidad ello no es así. El eje económico sigue siendo, precisamente, el crecimiento de las exportaciones, uno de los pilares de la política neoliberal (junto con el superávit fiscal). Además, tampoco parece que sintetice la voluntad estatal de buscar nuevas fórmulas para alcanzar el desarrollo, como podría inferirse del análisis de la iniciativa IIRSA.

La agenda de desarrollo e inclusión social en el nuevo regionalismo, es central y no parece estar comprendida en la calificación post-neoliberal. Es oportuno, entonces, considerar la idea de definirlo como "neo-estructural". El objetivo principal de este enfoque, al igual que en el estructuralismo, es el desarrollo de los países de la región, pero el mecanismo a través del cual se propone son las exportaciones (Leiva, 2008 y CEPAL, 2007), no la sustitución de importaciones (Valle, 2011). Desde este enfoque la intervención del Estado tiene un rol determinante, no para suplantar a las fuerzas del mercado, sino para estimular selectivamente las áreas capaces de contagiar dinamismo $y$ también para velar por el crecimiento con inclusión social y equidad. En ese sentido, el neo-estructuralismo también es complementario del neoliberalismo ${ }^{8}$.

Planteadas así las cosas, se piensa que la cuestión aún merece ser discutida. El "regionalismo post-neoliberal" es aún un concepto en construcción que, creemos, tal vez no sea el que represente mejor la síntesis del nuevo regionalismo sudamericano ${ }^{9}$. En efecto, existen

8 Leiva hace un análisis teórico del neo-estructuralismo, en el cual identifica dos rupturas con el estructuralismo: la idea de centro y periferia y la de la plusvalía apropiada por los países desarrollados, a partir de las materias primas provenientes de los países periféricos y luego vendidos en la periferia. notas inéditas que distinguen al fenómeno en cuestión; sin embargo, no son comprendidas en esa definición.

\section{UNASUR COMO PROYECTO POST- HEGEMÓNICO}

Hasta este punto se ha considerado la agenda de desarrollo y el fomento de las exportaciones como datos distintivos de la integración sudamericana. No obstante, se deben considerar otros elementos que hacen que ese proceso sea único en su tipo; es decir, al hecho de avanzar sobre temas que hasta entonces eran resueltos conforme los intereses del hegemón y a tratarlos sin su participación. Esta es una situación sin precedentes, ya que la influencia de EEUU en América Latina siempre ha ido en ascenso al punto que, durante la Guerra Fría, asfixiaba cualquier propuesta que se alejara de sus intereses.

Esa avanzada es particularmente notoria en la creación de un sistema sudamericano de reglas de integración física, de defensa, de constitución de un sistema financiero sudamericano y en menor medida, de la problemática de las drogas y la conformación de una instancia de observación electoral. Muchos de esos temas eran resueltos en el seno de la OEA (Organización de Estados Americanos) y siempre con la participación de EEUU. La avanzada también se observa en asuntos comerciales, donde la estrategia de regulación del hegemón es más compleja, combinando la acción hemisférica con acercamientos subregionales $y$ bilaterales.

Esto es lo que ha llevado a algunos autores a calificar al proceso en cuestión como "post-hegemónico" (Riggirozzi et ál., 2012). El dato distintivo no sería, entonces, la adopción de nuevas reglas distintas al US market-driven proposal (con la incorporación de las temáticas del desarrollo y la exclusión social), sino la "repolitización de la región" en función del

ción en el marco de nuestra tesis de maestría, se inclina por el concepto de integración "post-liberal de agenda ampliada", con miras a combinar la idea de complementariedad de la integración de los 90, con la agenda del desarrollo. 
aprovechamiento de los nuevos márgenes de maniobra internacionales (Kappel, 2011) para llevar adelante nuevos proyectos regionales. En términos de Dabène (2010), se estaría en presencia de la "politización fundacional" de la integración sudamericana que garantizaría su dinamismo.

El fin de la Guerra Fría trajo consigo un repliegue de EEuU en América Latina - situación que se acentuó con la guerra contra el terrorismo- y ello creó los incentivos para que la región actuara de manera más autónoma. A nivel mundial, ello dio lugar a la aparición de las nuevas potencias del Sur y cierto espacio para su acción en los respectivos ámbitos regionales (Nolte, 2006: 14 y Hurrell, 2007: 136-137). En el caso de América Latina, ese papel lo asumió Brasil que, a través de la regionalización de su "liderazgo consensual"10 (Burges, 2008: 65), busca posicionarse como referente de América del Sur y vocero del multilateralismo del Sur por un orden internacional más justo (Hofmeister, 2003; Gratius, 2007: 5 y Vigevani, 2009).

Sin ser potencias regionales, otros estados también aprovecharon esa permisividad internacional para llevar adelante sus propios proyectos regionales. Es el caso de Venezuela, la cual internacionalizó su proyecto alternativo de prédica anti-neoliberal en lo económico $y$ anti-hegemónico en lo político (Altmann, 2011: 186 y Sanjuán, 2008: 145). Su objetivo es un mundo equilibrado, respetuoso de la autodeterminación, la no intervención y con la solidaridad como fundamento de las relaciones internacionales (González, 2006: 161 y Briceño, 2011: 26-27).

El caso de Venezuela sirve para comparar con el proyecto político de México, único estado latinoamericano en condiciones de disputarle el liderazgo a Brasil. A pesar de también estar en ascenso (Hakim, 2002: 148), decidió no servirse de los nuevos márgenes de maniobra para ganar influencia en América Latina, sino prio-

$10 \quad$ Este tipo de liderazgo implica que la supremacía no se adquiere por el poderío militar o económico, sino gracias al consenso entre los demás estados de la idea-proyecto que define a uno de ellos como líder. Para ello, este debe atender los intereses de los estados seguidores, de modo de convencerlos de los beneficios de su liderazgo y del proyecto. rizar su inserción con América del Norte (González, 2008: 122) ${ }^{11}$. Sin México en competencia, Brasil encontró el camino allanado para consolidarse como líder de la región, aunque debió redefinir los límites de la misma. Una premisa de la política exterior brasileña ha sido evitar la confrontación directa con EEUU, preservando la relación preferencial cultivada desde la Segunda Guerra Mundial. La presencia del hegemón en México, en América Central y en el Caribe era demasiado importante como para la construcción de su liderazgo. De allí que limitara su proyección a América del Sur.

Así, con una clara definición de a quién se incluye $y$ a quién se excluye, América del Sur se ha ido constituyendo como ámbito (distinto al latinoamericano), desde el cual la región opera en el sistema internacional. Por eso, se sostiene junto a Riggirozzi y Tussie (2012), que el regionalismo dejó de ser la coordenada subordinada de la globalización económicafinanciera. La conformación de la Región trae consigo la construcción de su poder y el de su referente. De allí que no sea posible comprender el proceso sudamericano sin considerar la acción determinante de Brasil (Falomir, 2011).

Esa construcción de Sudamérica como región con su propio sistema de reglas es particularmente notoria en el tema de defensa. Su máxima expresión es el Consejo de Defensa Sudamericano (CDS) que, en tanto "instancia de consulta, cooperación y coordinación" (UNASUR, 2008), representa el núcleo de ese régimen. Sus metas son la preservación de la estabilidad en América del Sur - en tanto Zona de Paz-y la formación de una visión sudamericana de defensa para identificar amenazas $y$ riesgos, $y$ articular una posición común en los foros internacionales. Se destaca que el CDS no contempló la creación de una alianza militar, como pretendía Venezuela. En cambio, sí se incluyó la integración de las industrias de defensa con miras a alcanzar el autoabastecimiento y autono-

11 México padece un estado de "confusión estratégica" (González, 2008) por el fracaso de su plan de inserción dual: hacia el Norte con el TLCAN para fomentar su desarrollo $y$ hacia el Sur para hacer valer su autonomía. El fracaso se debió a que el plan solo fue exitoso en la primera dirección. 
mía (Moreira, 2009: 7); un objetivo prioritario de Brasil según su Plan Estratégico de Defensa (Ministerio de Defensa, 2008).

Íntimamente vinculado al tema de defensa y como nota absolutamente inédita, se debe mencionar la creación del Centro de Estudios Estratégicos de la Defensa del CDS (CEED-CDS): una instancia de elaboración de una doctrina de defensa propia. Ello trae consigo una ruptura con la lógica de formación del personal militar según la visión de la Junta Interamericana de Defensa, que funciona en el marco de la OEA. Resulta claro, entonces, el esfuerzo por generar la "identidad suramericana en materia de Defensa" con identificación de los propios desafíos, oportunidades y escenarios relevantes para la defensa y la seguridad regional y mundial (CEED, 2010) ${ }^{12}$.

En materia económica, el sistema de reglas de la UNASUR también se aparta de los lineamientos propuestos por EEUU. La concepción de Sudamérica como espacio geo-económicamente integrado, puesto en práctica a través de la iniciativa IIRSA (con sus ejes transnacionales de integración de infraestructura $y$ cadenas productivas), la adopción del PSCI como programa modelo de promoción de exportaciones del subcontinente $y$ la formación de un área sudamericana de comercio preferencial a partir de la convergencia CAN-Mercosur, dan cuenta de un alejamiento del modelo integrador de libre dirección del mercado, propiciado por EEUU. Si bien, Brasil será quien mayor provecho obtenga $^{13}$, es cierto que se trata de un esquema en el que se promueve (y necesita) el desarrollo de toda Sudamérica.

La propuesta de creación de un Sistema Financiero Sudamericano es otra muestra de la avanzada. En particular, la propuesta de utilizar monedas locales como medio de pago en el

12 Además del CDS, se crearon otros consejos temáticos referentes a: salud, energía, infraestructura y planeamiento, el problema mundial de las drogas, educación, ciencia y tecnología, economía y finanzas, desarrollo social y electoral.

13 El beneficio de la IIRSA para Brasil, radica en la posibilidad de acceso rápido y barato a los mercados de sus vecinos y las salidas a la cuenca del Pacífico, a través de los corredores bioceánicos. intercambio entre sudamericanos, suplantando al dólar estadounidense. Ello no solo abarata los costos de transacción sino que reduce la injerencia de EEUU por el uso de su moneda (algo oportuno en un contexto de crisis financiera internacional). En ese mismo sentido, también se señalan las propuestas de reducción de la vulnerabilidad financiera de Sudamérica con un Mercado de Deuda Regional y un Fondo de Estabilización Macroeconómica (o la reformulación del Fondo Latinoamericano de Reservas).

Sin embargo, la propuesta más osada de autorregulación fue la de Venezuela, la cual cosistió en de crear el Banco del Sur como alternativa al FMI; según Chávez: un "FMI para Latinoamérica" (ABN, 22/10/2007). Por ello, se destacan dos cuestiones al respecto: la primera, refiere a su proposición, que deja a la vista la inconveniencia del orden financiero internacional para los países de la región y la posibilidad de discutirlo. La segunda, refiere a la función asignada: a pesar de la intención de Chávez de crear un régimen divorciado del sistema financiero mundial, el Banco del Sur terminó siendo un banco de desarrollo, como pretendía Brasil, cuyos únicos beneficiarios pueden ser los países sudamericanos (Bacaria, 2011). Ello, como resultado de la cordial contienda por el liderazgo regional entre Venezuela y Brasil, con inclinación en favor de este último. Brasil logró, manteniendo la participación de Venezuela en su proyecto regional, reconvertir sus iniciativas en propuestas compatibles con sus intereses regionales; el Banco del Sur y el CDS son ejemplos de ello.

Otra área comprendida por esta integración inédita de autorregulación, es la gestión y contención de crisis políticas. Hasta entonces, tales cuestiones se resolvían en la OEA. Sin embargo, la CSN-UNASUR se mostró muy expeditiva para encausar distintas situaciones de crisis político-institucional como la de Ecuador en 2005, que concluyó con la salida de Lucio Gutiérrez de la Presidencia; o la disputa por la incursión del ejército colombiano en territorio ecuatoriano en marzo de 2008, para atacar un campamento de las FARC. Asimismo con la crisis de Bolivia de julio a noviembre de 2008, por 
el intento de escisión de la "media luna"; la discusión por la utilización de siete bases militares colombianas por EEUU en 2009; o el intento de golpe de estado en Ecuador en septiembre de 2010. Recientemente, volvió a mostrar sus reflejos ante un juicio político exprés en Paraguay.

Ese tratamiento prueba que el proceso sudamericano ha derivado en un verdadero "subsistema político internacional diferenciado" (Peña, 2009: 46). Se advierte, además, que la UNASUR ha reemplazado a la OEA en esos temas, lo que importa una limitante a las posibilidades de incidencia de EEUU en la región. Por los recientes desarrollos sudamericanos, esto podría ser extensible al problema de la droga (encarado desde una perspectiva no prohibicionista) y del crimen organizado trasnacional.

Por otra parte, así como se organizó cierta cohesión política hacia el interior del espacio sudamericano, también se definieron marcos de acción colectiva hacia el exterior de la región. En efecto, desde la creación misma de la CSN en Cuzco de 2004, se estableció como objetivo explotar la potencialidad aún no aprovechada de la región para fortalecer las capacidades de negociación y proyección internacionales (CSN, 2004, punto I). De allí los espacios de diálogo, bajo la modalidad de cumbres, generados con otras regiones del mundo en desarrollo, como con los Países Árabes (Cumbre ASPA) y África (Cumbre ASA). Más aún, durante la II Cumbre ASPA, en Qatar en marzo de 2009, América del Sur tuvo una sola voz representada por Chile, en ejercicio de la Secretaría Pro-témpore de la UNASUR.

\section{NUESTRA CARACTERIZACIÓN DE LA UNASUR: EL REGIONALISMO SUDAMERICANO}

A modo de cierre, es relevante aportar nuestra mirada sobre el proceso de integración de la UNASUR. Es evidente que las fórmulas explicativas de la integración de los años 90, no le son aplicables, aún cuando contemple la formación de un área de libre comercio en Sudamérica. Sin embargo, se cree que las dos caracterizaciones aquí analizadas no logran síntesis acabada del fenómeno. La calificación de post-neoliberal recoge acertadamente la importancia de la inserción en la economía mundial a través de las exportaciones. A la vez, deja entrever que comprende la agenda del desarrollo, una cuestión central en esta nueva fase del regionalismo. A pesar de ello, no es más explicativa que la del regionalismo neo-estructural. Pero ninguna de las dos explica la avanzada sobre los temas que antes eran regulados en función de los intereses del hegemón.

La calificación como post-hegemónica es, en ese sentido, superadora de las anteriores ya que ilustra el aprovechamiento del repliegue relativo de EEUU. Asimismo, refleja la situación inédita de un regionalismo "ofensivo", que pugna por su propia gobernanza y que define las reglas de interacción, tanto al interior de la región como hacia el exterior, en relación con otras regiones y actores. Sin perjuicio de ello, se entiende que la calificación adolece de un problema. Aún cuando el poder de EEuU no sea el de los años 90 y China se encuentre en un innegable ascenso (principalmente económico) y haya una mayor permisividad internacional, en el orden internacional actual, todavía no se observa una situación post-hegemónica. Más aún, mientras EEUU siga siendo líder en términos de desarrollo tecnológico y de conocimiento, con su consecuente impacto en lo cultural, económico y militar, seguirá siendo una potencia mundial. Con ello no se pretende obviar la indiscutible declinación relativa de poder del hegemón, pero sí señalar que el calificativo de post-hegemónico nos resulta un tanto pretensioso, ya que no se corresponde con la situación vigente en el orden internacional.

Por ello, nuestra propuesta es definir el fenómeno representado por la UNASUR como "Regionalismo Sudamericano". Así se hace referencia a la conformación de América del Sur como nueva región, cuya principal dimensión de integración es la política; un dato ajeno a las experiencias de integración latinoamericanas previas. Además, permite analizar el proceso desde la influencia de la Potencia Regional, Brasil, cuyo liderazgo consensual ha sido decisivo en las prácticas y normas sudamericanas. De allí la formación de un sistema sudamericano 
(de integración física, desarrollo, democracia, defensa, vulnerabilidad financiera, etc.) construido en torno a cuestiones que no solo le interesan al gigante sudamericano, sino también a sus vecinos.

En esa línea argumental, cabe aclarar que el carácter "ofensivo" es inherente a la idea de este tipo de regionalismo. Ello explica que en los inicios de la UNASUR, se hayan receptado los reclamos históricos de los latinoamericanos de un orden internacional más justo y multilateral. Así, la región se ha convertido en el vehículo por el que, aprovechando la mayor permisividad, se canalizan esas pretensiones de cambio. Asimismo, Brasil en tanto referente de tal espacio, se ve compelido a mostrarse como promotor de tales cambios, pero velando porque la orientación del novel sistema no se aparte de sus intereses regionales. Las propuestas del Banco del Sur, de la iniciativa IIRSA, del CDS y las acciones para promover y proteger la democracia, dan muestra acabada de ello. Por eso podríamos preguntarnos si Brasil no estaría promoviendo su propio regionalismo hegemónico.

\section{BIBLIOGRAFÍA}

LIBROS

Altmann, Josette. América Latina y el Caribe: ALBA. ¿Una nueva forma de integración regional? 1ra. Edición. Argentina: TeseoFLACSO-Fundación Carolina-OIRLA, 2011.

Bacaria Colom, Jordi. "Fines integracionistas y posibilidades financieras del Banco del Sur". Una región en construcción. UNASUR y la integración de América del Sur. Manuel Cienfuegos y Juan Antonio Sanahuja (editores). España: CIDOB, 2011.

Bouzas, Roberto; Da Motta Veiga, Pedro y Ríos, Sandra. "Crisis y perspectivas de la integración en América del Sur". América Latina: ¿integración o fragmentación? Ricardo Lagos (comp.). Argentina. Edhasa, 2008: 319-347.

Briceño Ruiz, José. "El ALBA como propuesta de integración regional". América Latina y el Caribe: ALBA: ¿Una nueva forma de integración regional? Josette Altmann (ed.). 1ra. Edición. Argentina: Teseo-
FLACSO-Fundación Carolina-OIRLA, 2011. Carranza, Mario Esteban. South American Free Trade Area or Free Trade Area of the Americas? Open regionalism and the future of Regional Economic Integration in South America. Estados Unidos: Ashgate, 2000.

CEPAL. Panorama de la inserción internacional de América Latina y el Caribe 20022003. Chile: CEPAL, mayo 2004.

CEPAL. Economic growth with equity. Challenges for Latin America. Estados Unidos: Palgrave Macmillan, 2007: 252.

González González, Guadalupe. "México en América Latina: entre el Norte y el Sur y el difícil juego del equilibrista”. América Latina: ¿integración o fragmentación? Ricardo Lagos (comp.). Argentina: Edhasa, 2008.

Hofmeister, Wilhelm. Brasil y sus vecinos: en búsqueda del liderazgo regional en América del Sur. Brasil. Konrad Adenauer Stiftung/PEE-UFRJ, 2003: 72.

Hurrell, Andrew. Regionalism in world politics. Regional organization and international order. Gran Bretaña. Oxford University Press, 1995: 342.

Leiva Letelier, Fernando Ignacio. Latin American Neo-structuralism: the contradiction of Post-neoliberal development. Estados Unidos. University of Minesota Press, 2008: 312.

León, José Luis. "El orden mundial de la posguerra fría". Política internacional contemporánea. Zidane Zeraoui (dir.). México. Trillas, 2000: 225-256.

Macdonald, Laura y Rückert, Arne. Postneoliberalism in the Americas. Estados Unidos. Palgrave Macmillan, 2009: 296.

Panizza, Francisco. Contemporary Latin America: development and democracy beyond the Washington Consensus. 1ra. Edición. Gran Bretaña: Zed Books, 2009.

Prebisch, Raúl. Desarrollo económico en América Latina y sus principales problemas. Chile: CEPAL, 1948.

Riggirozzi, Pia y Tussie, Diana. "The rise of Post-hegemonic regionalism: the case of Latin America". United Nations 
University Series on Regionalism 4. 1ra. Edición. Estados Unidos: Springer, 2012.

Smith, Peter. Talons of the eagle: dynamics of US-Latin American relations. Gran Bretaña. Oxford University Press, 2000: 418.

Sanjuán, Ana María. "América Latina y el bolivarismo del Siglo xxi. Alcances $y$ desafíos de la política venezolana hacia la región". América Latina: cintegración o fragmentación? Ricardo Lagos (comp.). Argentina: Edhasa, 2008.

Soares De Lima, Maria Regina. "El lugar de América del Sur en la política externa brasileña". Obras de integración física en América del Sur. República Federal de Brasil-Ministerio de Relaciones Exteriores, 2008.

Stiglitz, Joseph E. (2002). El malestar en la globalización. 2da. Edición. Argentina: Taurus-Alfaragua Ediciones SA, 2006.

REVISTAS

Burges, Sean W. "Consensual hegemony: theorizing Brazilian foreign policy after the Cold War". International Relations 22 (1). 2008: 65-84.

González Urrutia, Edmundo. "Las dos etapas de la política exterior de Chávez". Nueva Sociedad 205. Fundación Friedrich Ebert, septiembre-octubre 2006: 159-171.

Grugel, Jean y Rigirozzi, Pia. "Postneoliberalism in Latin America: rebuilding and reclaiming the state after crisis". Development and Change 43 (1). 2012: 1-21.

Haas, Ernest B. "The United States of Europe". Political Science Quarterly LXX (4). Diciembre 1948.

Hakim, Peter. "Two ways to go global. The differing paths of Mexico and Brazil". Foreign Affairs 81 (1). Enero-febrero 2002: 148-162.

Hurrell, Andrew. "One world? Many worlds? The place of regions in the study of international society". International Affairs 83 (1). 2007: 127-146.

Kacowicz, Arie. "América Latina en el mundo: globalización, regionalización y fragmentación”. Nueva Sociedad 214. Fundación Friedrich Ebert, marzo-abril 2008.

Lagos, Marta y Zovatto, Daniel. "Mitos $y$ realidades de la integración latinoamericana". Foreign Affairs (versión en español). Octubre-diciembre 2007.

Lowenthal, Abraham. "De la hegemonía regional a las relaciones bilaterales complejas: Estados Unidos y América Latina a principios del Siglo XxI". Nueva Sociedad 206. Fundación Friedrich Ebert, noviembre-diciembre 2006.

Mallmann, Maria Izabel. "Límites y perspectivas de la integración sudamericana". Cuadernos sobre Relaciones Internacionales, Regionalismo y Desarrollo 2 (4). Juliodiciembre 2007: 39-67.

Nolte, Detlef. "Potencias regionales en la política internacional: conceptos y enfoques del análisis". GIGA Working Paper 30. Hamburgo, 2006: 37.

Molano Cruz, Giovanni. "La integración andina: origen, transformaciones $y$ estructuras". Integración \& Comercio 33. Año 15. InTAL. Julio-diciembre 2011.

Peña, Felix. "¿La UnASUR y el MERCOSUR pueden complementarse?". Nueva Sociedad 219. Fundación Friedrich Ebert, 2009.

Rojas Aravena, Francisco. "El nuevo mapa político latinoamericano. Para repensar los factores que marcan las tendencias políticas". Nueva Sociedad 215. Fundación Friedrich Ebert, 2006.

Rojas Aravena, Francisco. "América Latina y los desafíos para la integración regional". Anuario CEIPAZ 2. 2008-2009: 99-120.

Rojas Aravena, Francisco. "La integración regional: un proyecto político estratégico". III Informe del Secretario General de FLACSO. Costa Rica: FLACSO, 2008.

Rovira Katwasser, Cristobal. "Towards Postneoliberal in Latin America". Latin American Research Review 46 (2). 2011: 225-234. 
Sanahuja, José Antonio. "Regionalismo e integración en América Latina: balance y perspectivas". Pensamiento Iberoamericano 2 . Instituto Complutense de Estudios Internacionales, 2006.

Sanahuja, José Antonio. "Del regionalismo abierto al regionalismo post-liberal. Crisis y cambio en la integración regional en América Latina". Anuario de la integración regional de América Latina y el Caribe 7. Buenos Aires: CRIES, 2008-2009.

Sanahuja, José Antonio. "Multilateralismo y regionalismo en clave suramericana: el caso de UNASUR". Pensamiento propio: los desafíos del multilateralismo en América Latina 33. Año 16. Edición especial. Buenos Aires. CRIES-Universidad de GualadajaraUniversidad Iberoamericana. Enero-julio 2011: 115-156.

Tokatlian, Juan Carlos. "La unión sudamericana: ¿inexorable o contingente?". Desarrollo Económico 41 (161). Abril-junio 2001.

Valle Pérez, Francisca. "Un acercamiento teórico al neoestructuralismo en América Latina". Anuario 2011 Facultad de Ciencias Económicas y Empresariales. Santiago de Cuba. Universidad de Oriente, 2011: 58-66.

Vigevani, Tullo y Ramanzini, Haroldo Jr. "Brasil en el centro de la integración". Nueva Sociedad 219. Fundación Friedrich Ebert, 2009.

TESIS

Falomir Lockhart, Nicolás. "Integración sudamericana. La influencia de los proyectos políticos de Brasil y Venezuela" [Tesis de Maestría en Relaciones Internacionales] Universidad Nacional de La Plata (UNLP), 2011.

\section{TEXTOS ELECTRÓNICOS}

Álvarez Valdéz, Rodrigo. "UNASUR: desde la perspectiva subregional a la regional". Serie Documentos Electrónicos 6.
FLACSO-Chile. Octubre 2009. En: <http:// www.flacso.cl $>$ [consultado el 20 de marzo de 2012].

CEPAL. "El regionalismo abierto en América Latina y el Caribe. La integración económica al servicio de la transformación productiva con equidad". Libros de la CEPAL 39. Chile. Enero 1994. En: <http://www.eclac.cl/publicaciones/ $\mathrm{xml} / 7 / 4377 / \operatorname{lcg} 1801 \mathrm{e} . \mathrm{htm}>$ [consultado el 21 de marzo de 2012].

Dabène, Olivier. "Consistency despite instability, resilience despite crises. Explaining Latin American regional integration's oxymoron". Working Paper 121. FlaCso. Junio 2010. En: <http:// rrii.flacso.org.ar/?page_id $=1940>$ [consultado el 2 de marzo de 2012].

Gratius, Susanne. "Brasil en las Américas: ¿una potencia regional pacificadora?”. FRIDE Documento de Trabajo 35. Abril 2007. En: <http://www.fride.org/ publicacion/223/brasil-en-las-americasuna-potencia-regional-pacificadora $>$ [consultado el 2 de abril de 2012].

Kappel, Robert. "The decline of Europe and the US: shifts in the world economy and in global politics". GIGA Focus International 1. 2011. En: <www.giga-hamburg.de/> [consultado el 15 de abril de 2012].

Moreira, Ángela. "Consejo Sudamericano de Defensa: hacia una integración regional en defensa". Red de Seguridad y Defensa de América Latina-RESDAL. 2009. En: $<$ http://www.resdal.org > [consultado el 20 de abril de 2012].

Soares De Lima, Maria Regina. "Integracao moderna”. Análise de Coyuntura OPSA 1. Observatótio Político Sul-Americano-IUPRJ, 2006. En: <http://observatorio.iesp.uerj.br> [consultado el 21 de mayo de 2012].

OTROS

Agencia Bolivariana de Noticias (ABN). "Venezuela impulsa nueva arquitectura monetaria latinoamericana". 22 de octubre de 2007. Tomado del Portal Aporrea.org. En: <http://www. alternativabolivariana.org/modules.php 
?name $=$ News \&file $=$ article $\&$ sid $=2402>$ [consultado el 20 de febrero de 2011].

Banco del Sur. Convenio constitutivo del Banco del Sur. 26 de junio. Isla Margarita, República Bolivariana de Venezuela, 2009.

Centro de Estudios Estratégicos de Defensa (CEED-CDS). Estatuto del Centro de Estudios Estratégicos de Defensa del CDS. 2010. En: <http://www.unasursg.org> [consultado el 16 de abril de 2012].

Comunidad Sudamericana de Naciones (CSN). "Declaración de Cusco sobre la Comunidad Sudamericana de Naciones". Cusco 8. Diciembre 2004.

Consejo de Desarrollo Social Sudamericano. Estatuto del Consejo de Desarrollo Social Sudamericano. UNASUR. 10 de agosto 2009.

Iniciativa para la Integración de la Infraestructura Regional de
Sudamérica (IIRSA). En: <http:// www.iirsa.org $>$ [consultado el 07 de mayo de 2012].

Partido Social Cristiano-República Federativa del Brasil. 2011. En: <http://www. brasilglobalnet.gov.br/frmprincipal.aspx> [consultado el 14 de abril de 2012].

Ministerio de Defensa del Brasil. Plan Estratégico de Defensa. 2008. En: $<$ http://www.defesa.gov.br/projetosweb/ estrategia/> [consultado el 16 de abril de 2012].

UNASUR. Declaración de Costa do Sauipe sobre la creación del Consejo de Defensa Sudamericano. Brasil. 16 de diciembre 2008. En: <http://www.resdal.org/csd> [consultado el 25 de mayo de 2011].

Fecha de ingreso: 13/07/2012 Fecha de aprobación: 25/09/2012 
